\title{
Chemical signatures of shocks in hot cores
}

\author{
S. Viti ${ }^{1}$, P. Caselli ${ }^{2}$, T. W. Hartquist ${ }^{3}$, and D. A. Williams ${ }^{1}$ \\ 1 Department of Physics and Astronomy, University College London, London WC1E 6BT, UK \\ 2 Osservatorio Astrofisico di Arcetri, Largo E. Fermi 5, 50125 Firenze, Italy \\ 3 Department of Physics and Astronomy, University of Leeds, Leeds, LS2 9JT, UK
}

Received 4 August 1999 / Accepted 26 February 2001

\begin{abstract}
The characteristic chemistry of hot cores arises from the abrupt evaporation of icy mantles when a massive star begins to irradiate the interstellar gas in its vicinity. Such stars are also likely to generate powerful winds which may initiate shocks in the same interstellar gas. In this paper, we consider whether chemical signatures of the passage of a shock through a hot core can be identified. We find that if hydrogenation occurs on surfaces and freeze-out of heavy gas-phase atoms and molecules is complete before the hot core is established then no such chemical signature exists. However, if some residual material is present in the gas when the hot core is established then the following molecular abundance ratios are significantly affected by the presence of a shock: $\mathrm{NS} / \mathrm{CS}, \mathrm{SO} / \mathrm{CS}$, and $\mathrm{HCO} / \mathrm{H}_{2} \mathrm{CO}$. This result is more evident if injection of ices into the gas occurs over a finite period, rather than instantaneously. We conclude that these molecular abundance ratios may be useful tracers of the dynamical history of hot cores, and that follow-up observational studies are required.
\end{abstract}

Key words. stars: formation - ISM: abundances - molecules - shock waves

\section{Introduction}

Hot cores are dense, small, warm clumps of gas found close to young massive stars (see, e.g., reviews by Millar 1993; Walmsley \& Schilke 1993 and more recently Kurtz et al. 2000). They contain abundances of molecules that appear somewhat anomalous when compared with those in the much larger and cooler interstellar molecular clouds, being richer, for example, in saturated hydrocarbons. Hot core chemistry arises generally as a consequence of high mass star formation, although it has also been observed towards the low-mass protostar IRAS 16293 (Ceccarelli et al. 2000). In the case of massive star formation, the newly formed star heats dust in its vicinity, and the warmed dust then evaporates the icy mantles that were deposited in the pre-stellar phase. Thus, the molecules released into the gas trace directly the physical conditions during the cloud collapse that led to star formation.

There have been many studies of the chemical evolution of the molecules from the evaporated ices (e.g. Brown et al. 1988; Caselli et al. 1993; Charnley 1997; Millar et al. 1997; Cesaroni et al. 1998; Hatchell et al. 1998a; Millar \& Hatchell 1998; Viti \& Williams 1999). These have shown that observed hot cores must have ages limited to $10^{4}$ to $10^{5}$ years. Therefore, the study of hot cores gives insight

Send offprint requests to: S. Viti, e-mail: sv@star.ucl.ac.uk into events close in time as well as space to the formation of the star. For most models it is assumed that ice is evaporated from the dust instantaneously, immediately after the star has formed. However, Viti \& Williams (1999) noted that the star will not attain its maximum luminosity instantaneously, but over a finite period that may be comparable with the normal age of a hot core. The dust would in this case be warmed up over a comparable period, so that initially only weakly bound molecules such as $\mathrm{CO}$ and $\mathrm{N}_{2}$ would be released from the ice while more strongly bound molecules (e.g. $\mathrm{H}_{2} \mathrm{O}$ ) would be released later. In this view, the chemical composition of material entering the gas phase changes with time. Therefore, the contraction time of high mass stars to the main sequence (a poorly known quantity; Hanson 1998) might possibly be inferred from the time dependent chemical composition of hot cores.

However, this is not the only possible generalization of the commonly accepted picture of hot core evolution. In addition to their luminosities, young stars generate winds that may strongly influence the stellar environments. It is well known that low-mass pre-zero age main sequence (ZAMS) stars have strong winds (Bachiller 1996). Given the present lack of knowledge about high mass stars at a similar stage of evolution, we assume that they also may have winds with mechanical luminosities of the 
order of $10 L_{\odot}$ (Shepherd \& Churchwell 1996). This view is supported by observations that show the existence of outflows around stars without UCHII regions (Reid et al. 1995; Shepherd \& Churchwell 1996; Molinari et al. 1998; Cesaroni et al. 1999). We therefore consider the possible consequences of winds from the star impacting on the material that will become the hot core, and address the following question: if wind-generated shocks desorb molecules from ices, are there any observational tracers of this effect? We shall assume that the shock may arrive on the core gas before, during or after the thermal evaporation process driven by the stellar radiation.

Although the stellar wind is likely to have a very high velocity (much larger than $100 \mathrm{~km} \mathrm{~s}^{-1}$ ) as it leaves the star, the perturbation it creates in the dense hot core travels with a much smaller velocity. In this paper, we consider the effects of relatively slow $\mathrm{C}$-type shocks, with velocities around 10 to $20 \mathrm{~km} \mathrm{~s}^{-1}$. Shocks of much higher speed would be easily traceable through $\mathrm{SiO}$ emission (Lada et al. 1978) from sputtered grains. Shocks of much smaller speeds than $10 \mathrm{~km} \mathrm{~s}^{-1}$ are unlikely to make a significant contribution to the chemistry in hot cores as the postshock temperatures would be too low to affect the chemistry significantly. We are making the first detailed study of the possible effects of shocks that may leave subtle evidence of their existence within hot cores. If this initial study indicates that shock tracers can be identified, then future studies will have to develop a detailed treatment of the shock passage. We consider only the effect of the passage of a single shock. It is possible to envisage that the wind may vary in such a way as to generate a succession of shocks. However, a steady wind acting on a smooth core will send only a single shock through it, and this is the system that we explore in this initial calculation. Multiple shocks are beyond the scope of the present calculation.

In Sect. 2 we describe the details of the models evaluated, and in Sects. 3, 4, and 5 we give results for several different classes of model. Section 6 contains our conclusions from this first detailed study of this complex problem. These conclusions are quite straightforward, and are given briefly here. Firstly, if all species more massive than helium are completely frozen-out so that none of them remains in the gas phase and hydrogenation occurs on surfaces, then we can find no chemical signature of the passage of a shock through the hot core, regardless of when the shock arrives in the core's evolution. Secondly, if as observations suggest - there is always a small residual amount of matter containing elements more massive than helium in the gas phase, then the following ratios of molecular abundances are specific indicators of shock passage: NS/CS; SO/CS; and $\mathrm{HCO} / \mathrm{H}_{2} \mathrm{CO}$. Thirdly, these ratios are useful indicators both in cases where the warming of the dust by stellar radiation takes place instantaneously and where it occurs over a finite period.

These clear conclusions highlight, however, the present paucity of suitable high resolution observations in lines of molecules that are appropriate for testing the shock hypothesis. The over-riding conclusion is, therefore, that further specific observations still need to be made if we are to assess whether dynamical as well as radiative effects are likely to be important within hot cores.

\section{The model}

We follow the chemical evolution in a cloud that is collapsing in a "modified" free-fall (Ruffle et al. 1999) in the process of forming a massive star. The collapse is ultimately arrested, and a star is assumed to be formed. Our calculations are space- and time-dependent as described in Viti \& Williams (1999) but with only five depth points of increasing visual extinction up to 620 mags, taken to be the collapse centre. The chemical evolution of the remnant core is explored during the collapse and after its arrest, including the passage of a shock. The molecular cloud is assumed to have initial and final densities of $10^{4} \mathrm{H}_{2} \mathrm{~cm}^{-3}$ and $10^{7} \mathrm{H}_{2} \mathrm{~cm}^{-3}$, respectively, and an initial and final visual extinction at the centre of the core of 1.2 and 620 mags, respectively. We have included 119 gas-phase species and 39 surface species interacting in 1729 chemical reactions taken from the UMIST data base (Millar et al. 1997). The surface reactions included in this calculation have been described by Viti \& Williams (1999), and are briefly as follows: It is assumed that all species that can be hydrogenated on dust grain surfaces will be fully saturated, e.g. $\mathrm{O}$ atoms are converted to $\mathrm{H}_{2} \mathrm{O}$ and $\mathrm{C}$ atoms to $\mathrm{CH}_{4}$, and retained on the surface. In addition, a small fraction of CO, 0.01 per cent (as suggested by models such as those of Charnley et al. 1992), is assumed to be converted to methanol on the surfaces of grains.

The initial elemental abundances by number relative to hydrogen were taken to be $0.14,4.010^{-4}, 1.010^{-4}$, $7.010^{-5}, 1.310^{-7}, 1.010^{-7}$ for helium, oxygen, carbon, nitrogen, sulphur and magnesium. Note that, as we start from a purely atomic chemistry (apart from hydrogen) after freeze out is completed, $\mathrm{NH}_{3}$ will contain much of the nitrogen. The sulphur abundance was chosen to be a factor of one hundred lower than solar (Oppenheimer \& Dalgarno 1974). The temperature is held at $10 \mathrm{~K}$ throughout the collapse. After the collapse phase, the temperature rises from the initial value of $10 \mathrm{~K}$ to a value of $226 \mathrm{~K}$ at the centre of the core. In the models explored here, this temperature rise may be instantaneous (as is conventionally assumed) or occur over a period of some tens of thousands of years (as discussed by Viti \& Williams 1999). The gas and dust temperatures (assumed to be the same because of the very high densities considered) also vary as a function of depth (Viti \& Williams 1999). Hot core kinetic temperatures range from $90 \mathrm{~K}$ to $300 \mathrm{~K}$, with a mean value of $\sim 170 \mathrm{~K}$ (Kurtz et al. 2000). Most the reactions included in our rate file and discussed in this paper are not significantly affected by the difference between our estimate and the mean value. Away from the centre of the core our temperature is higher, reaching $300 \mathrm{~K}$ at the edge $\left(A_{V}=3.6 \mathrm{mag}\right)$; if the source of heating is internal, rather than external, the material at a given temperature would be spatially distributed somewhat differently but a range 
of temperatures, similar to the ones considered here, would be present in either case. Rowan-Robinson (1980) derived an expression for the temperature distribution dependence on depth which we have fitted with an exponential expression and have extended to include a linear dependence on time (see Viti \& Williams 1999). However, in this paper we mainly present and discuss the evolution at the centre of the core. For a full discussion of the effect of grain evaporation through the core the reader is referred to Viti \& Williams (1999).

We have computed an extensive grid of models to investigate the effects of a shock on the chemistry of the remnant core. Results for selected models are listed in Tables 1 and 2 where we selected species shown to be good shock indicators. In this paper we will mainly discuss results shown in Table 1 (centre of the core) although results for a different depth point in the core where the temperature is $\sim 270 \mathrm{~K}$, are shown for completeness in Table 2 and will be briefly discussed. The shock is low velocity and its consequences are assumed to be two-fold: the removal of all mantles, and the increase of temperature to $1000 \mathrm{~K}$ for a period of 100 years, representing the temperature structure of a $\mathrm{C}$-shock. This temperature profile was adopted from the calculation of Bergin et al. (1998) who studied the chemistry of $\mathrm{H}_{2} \mathrm{O}$ and $\mathrm{O}_{2}$ in postshock gas. To designate the models, we use $\mathrm{T}$ and $\mathrm{S}$ to signify the time dependence of the radiative heating and the presence of the shock. The letter $\mathrm{T}$ is always followed by the time (measured in thousands of years) over which the grain temperature attains its maximum. Thus, T(70) means that the grains reach this temperature 70000 years after the star switches on. Instantaneous heating is represented by $\mathrm{T}(0)$. The shock may occur at any time during the warming period or after the grains attain their maximum temperature. Thus, $\mathrm{S}(0)$ implies that the shock passed at the moment when radiative heating by the star began. $\mathrm{S}(20)$ implies that the shock passed 20000 years after the onset of radiative heating. Finally, the models were run for different percentages of elemental carbon in all forms remaining in the gas at the end of the collapse phase; these percentages are written at the end of the model name. For instance, T(70)S(20)15\% is the model in which the radiative heating rate rises over a period of 70000 years, after freeze-out left $15 \%$ of the elemental carbon in gas phase in all forms, and in which a shock passed 20000 years after the onset of radiative heating.

\section{Simple models with total freeze-out and shocks}

The chemistry during the collapse is an entirely conventional interstellar chemistry (cf. van Dishoeck 1998) in which ion-molecule and neutral reactions create molecules of increasing complexity. Ultimately, freeze-out of atoms and molecules on to dust grains removes all species other than $\mathrm{H}_{2}$ and $\mathrm{He}$ from the gas. It is then assumed that the star switches on and that the core is heated by stellar radiation and possibly shocked by its wind. In this section, we compare the results of two models to explore the consequences of shock chemistry in the core.

For model $\mathrm{S}(0) \mathrm{T}(28) 0 \%$ a shock was assumed to sputter all mantle material and to maintain the core material at $1000 \mathrm{~K}$ for 100 years after which rapid cooling was assumed to occur; we supposed that the shock has a fastmode Mach number not greatly in excess of unity so that the density remained reasonably constant during and after its passage. After the post-shock cooling, freeze-out was assumed to occur at the same rate as during the initial freeze-out phase. Postshock freeze-out was assumed to be total. Then time dependent heating of mantle material was assumed to increase linearly over 28000 years.

Model $\mathrm{T}(28) 0 \%$ is of a core in which no shock, and therefore no second freeze-out, occurs. Otherwise the evolution is similar to that in model $\mathrm{S}(0) \mathrm{T}(28) 0 \%$; that is, time dependent grain heating to $200 \mathrm{~K}$ taking 28000 years is assumed.

Figure 1 gives results for some species of interest for model $\mathrm{S}(0) \mathrm{T}(28) 0 \%$. These are the species having results (displayed in Fig. 1a) depending significantly on whether or not a shock is incorporated in the models. The other species (results displayed in Fig. 1b) are those which are important in the chemical network, and an inspection of the time evolution of their abundances provides insight into why the abundances of a number of observable species behave as they do. Note that the fractional abundance of HCO does not reach a steady state until the fractional abundance of oxygen starts declining: in fact, the first rise of HCO corresponds to its evaporation from the grain; as soon as oxygen reaches its peak (at $\sim 6000$ yrs) $\mathrm{HCO}$ is efficiently destroyed, but it also continues to be efficiently formed by $\mathrm{H}_{2} \mathrm{CO}+\mathrm{O}$ and $\mathrm{H}_{2} \mathrm{CO}+\mathrm{OH}$ reactions. It finally reaches steady state at $\sim 25000$ yrs when the oxygen abundance starts falling steeply. In general, the nonsteady behaviour of many species at early times is due to the selective evaporation treatment. Table 1 gives values of abundance ratios at several times for both models $\mathrm{S}(0) \mathrm{T}(28) 0 \%$ and $\mathrm{T}(28) 0 \%$.

Table 1 shows that the time variation of abundances, after thermal evaporation begins, differs very little between the two models. This is a consequence of the fact that hydrogenation on grain surfaces has been supposed to occur, producing ices such as $\mathrm{H}_{2} \mathrm{O}, \mathrm{CH}_{4}, \mathrm{NH}_{3}$ and $\mathrm{H}_{2} \mathrm{~S}$. When these species return to the gas phase due to sputtering in a shock, the high temperature chemistry in the $\mathrm{H}_{2}$ rich gas does not remove them. Though the qualitative nature of the agreement between the results in Table 1 may not be totally unexpected, the rather precise quantitative agreement between the results from the two models is striking, and illustrates that the evaporated products of surface chemistry and of high temperature shock chemistry can be similar. Abundances of deuterated species may be also used as a way to differentiate between thermal evaporation and shock chemistry as they would be more efficiently destroyed in a shocked hot core. However, at temperatures of the order of $200 \mathrm{~K}$, deuterated species 
(a)

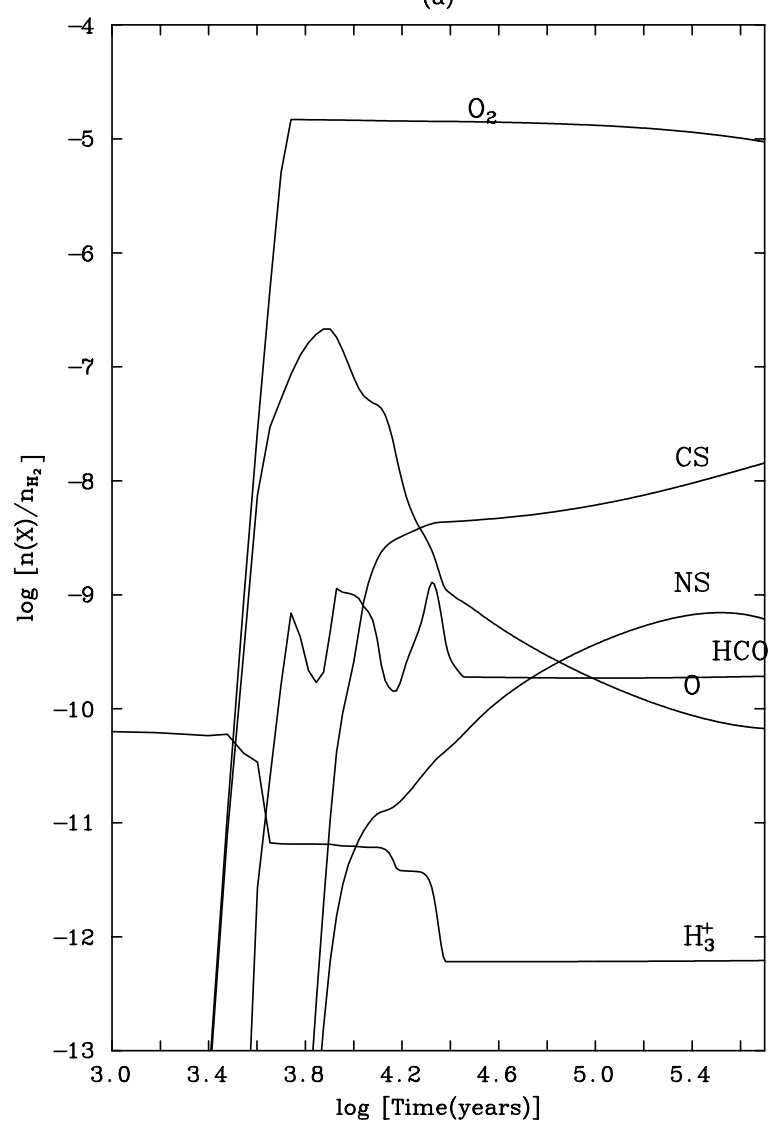

(b)

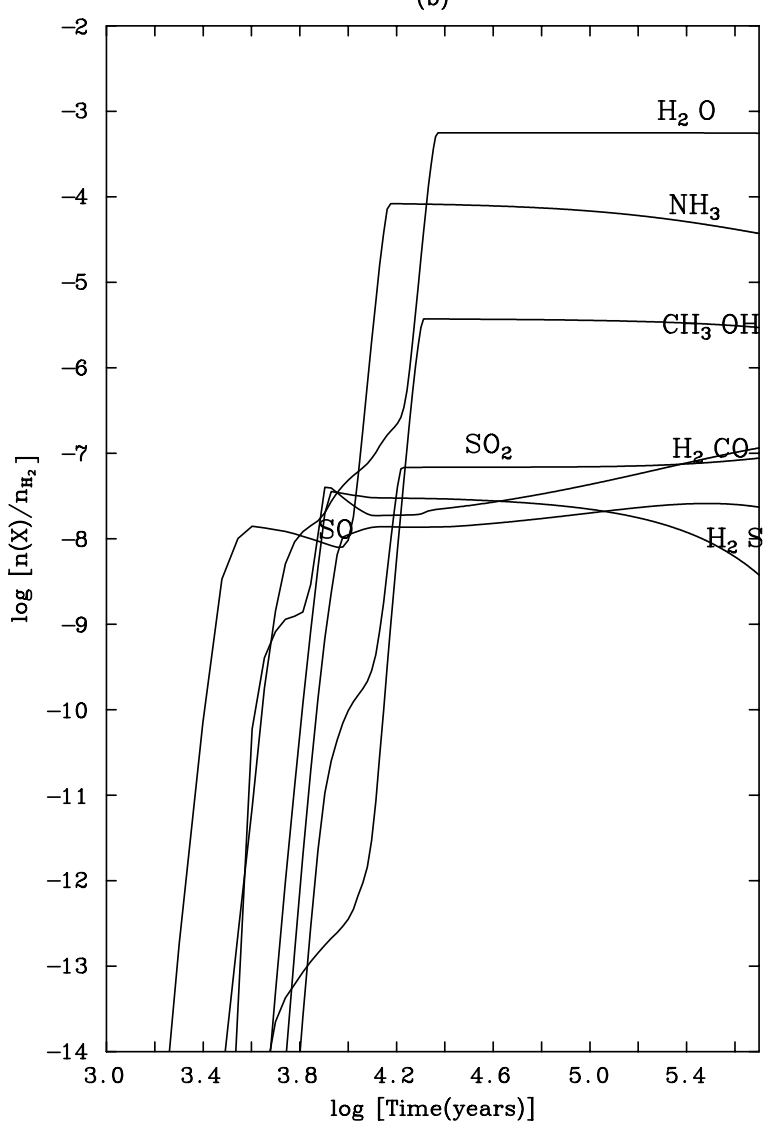

Fig. 1. Evolution of fractional abundances for $\mathrm{S}(0) \mathrm{T}(28) 0 \%$. The fractional abundances are relative to the number of hydrogen molecules. Time is measured from when the grain temperature increase, due to radiative heating, begins

would eventually be destroyed even if a shock has not occurred (Hatchell et al. 1998b); therefore the abundances of deuterated species may not be able to distinguish between a young shocked core and a more evolved thermally heated core unless other species are used as tracers.

Clearly, if hydrogenation is strongly dominant and freeze-out in the collapse and postshocked phases is total, observations of hot core chemistry will give no insight into the hot core dynamics, nor into the manner in which stellar winds turn on. This is the first important result of this study.

\section{Simple models with partial freeze-out and shocks}

Although a number of cold dense cores are known where very substantial depletion, by a factor of about 10, of gas phase CO obtains (e.g. Willacy et al. 1998; Caselli et al. 1999; Kramer et al. 1999), it is possible that freeze-out is never total, as a variety of desorption processes should operate even in cold, dark regions (e.g. Williams 1993). In cold dense cores observed in Orion a few percent of the elemental carbon is retained in the gas phase in CO (Gibb \& Little 1998). Sulphur is more highly depleted in many environments than other elements, but a few tenths of a percent or more of sulphur is always found in gas phase species within dense cores. Another explanation (cf. Ruffle et al. 1999) might account for some sulphur remaining in the gas phase even when it is highly depleted, but it is possible that a continuous desorption process may limit the sulphur depletion. Other studies of depletion (e.g. Bergin et al. 1997) always use some molecular tracers of the gas, implying that freeze-out on to dust is never total. We have taken the observations of Gibb \& Little as a guide to depletions in very dense cores, and have assumed that freezeout halts when a small fraction (3\% in most models) of the elemental carbon is left in gas phase species; a good fraction of the gas phase carbon is in $\mathrm{CO}$, but the halt in freeze-out also leaves similar fractional abundances of other more reactive species in the gas phase which can take part in shock-induced high-temperature chemistry. We explore the possibility that the passage of a shock through the core (which still contains at least some gas phase species) - either before it is warmed, or after - can be detected from the core's chemical composition.

In this section we present results for two simple models in which partial freeze-out has been assumed to take place. Model $\mathrm{S}(0) \mathrm{T}(0) 3 \%$ is for a core through which a shock passes after collapse has occurred; sputtering in the shock of all mantle material without subsequent freezeout was assumed (so that the focus was on the shock 
Table 1. Abundance ratios at three times (in years) for selected models at the centre of the core corresponding to $A_{V}=600$ mags and $T=220 \mathrm{~K}$

\begin{tabular}{|c|c|c|c|c|c|}
\hline & $\mathrm{HCO} / \mathrm{H}_{2} \mathrm{CO}$ & $\mathrm{SO} / \mathrm{CS}$ & $\mathrm{SO}_{2} / \mathrm{SO}$ & $\mathrm{H}_{2} \mathrm{~S} / \mathrm{CS}$ & $\mathrm{NS} / \mathrm{CS}$ \\
\hline \multicolumn{6}{|c|}{$2.510^{4}$} \\
\hline $\mathrm{T}(0) 3 \%$ & $0.53(-2)$ & $0.24(+2)$ & $0.14(+3)$ & $0.71(+2)$ & $0.17(+0)$ \\
\hline $\mathrm{T}(28) 15 \%$ & $0.29(-3)$ & $0.61(+4)$ & $0.47(+5)$ & $0.75(+4)$ & $0.48(-4)$ \\
\hline $\mathrm{T}(28) 0 \%$ & $0.11(-1)$ & $0.32(+1)$ & $0.16(+2)$ & $0.66(+1)$ & $0.10(-1)$ \\
\hline $\mathrm{T}(28) \mathrm{S}(10) 3 \%$ & $0.44(-1)$ & $0.68(+1)$ & $0.38(+2)$ & $0.18(+2)$ & $0.50(+0)$ \\
\hline $\mathrm{T}(28) \mathrm{S}(10) 15 \%$ & $0.56(-1)$ & $0.87(+1)$ & $0.12(+3)$ & $0.27(+2)$ & $0.30(+0)$ \\
\hline $\mathrm{T}(70) 3 \%$ & $0.37(-2)$ & $0.11(+2)$ & $0.64(+1)$ & $0.21(+2)$ & $0.31(-4)$ \\
\hline $\mathrm{T}(70) 15 \%$ & $0.69(-3)$ & $0.26(+2)$ & $0.82(+2)$ & $0.32(+2)$ & $0.30(-5)$ \\
\hline $\mathrm{T}(70) \mathrm{S}(20) 3 \%$ & $0.14(-1)$ & $0.57(+1)$ & $0.41(+2)$ & $0.21(+2)$ & $0.56(+0)$ \\
\hline $\mathrm{T}(70) \mathrm{S}(20) 15 \%$ & $0.37(-1)$ & $0.12(+2)$ & $0.21(+3)$ & $0.54(+2)$ & $0.74(-1)$ \\
\hline $\mathrm{T}(70) \mathrm{S}(50) 3 \%$ & \multicolumn{5}{|c|}{ Same as $\mathrm{T}(70) 3 \%$} \\
\hline $\mathrm{T}(70) \mathrm{S}(50) 15 \%$ & \multicolumn{5}{|c|}{ Same as $\mathrm{T}(70) 15 \%$} \\
\hline $\mathrm{S}(0) \mathrm{T}(0) 3 \%$ & $0.43(-2)$ & $0.38(+1)$ & $0.47(+2)$ & $0.26(+2)$ & $0.18(-1)$ \\
\hline $\mathrm{S}(0) \mathrm{T}(28) 0 \%$ & $0.12(-1)$ & $0.32(+1)$ & $0.16(+2)$ & $0.67(+1)$ & $0.10(-1)$ \\
\hline \multicolumn{6}{|c|}{$5.010^{4}$} \\
\hline $\mathrm{T}(0) 3 \%$ & $0.47(-2)$ & $0.13(+2)$ & $0.62(+2)$ & $0.29(+2)$ & $0.20(+0)$ \\
\hline $\mathrm{T}(28) 15 \%$ & $0.97(-3)$ & $0.82(+3)$ & $0.65(+4)$ & $0.81(+3)$ & $0.37(-3)$ \\
\hline $\mathrm{T}(28) 0 \%$ & $0.61(-2)$ & $0.34(+1)$ & $0.14(+2)$ & $0.54(+1)$ & $0.34(-1)$ \\
\hline $\mathrm{T}(28) \mathrm{S}(10) 3 \%$ & $0.36(-1)$ & $0.52(+1)$ & $0.28(+2)$ & $0.13(+2)$ & $0.57(+0)$ \\
\hline $\mathrm{T}(28) \mathrm{S}(10) 15 \%$ & $0.41(-1)$ & $0.53(+1)$ & $0.70(+2)$ & $0.16(+2)$ & $0.76(+0)$ \\
\hline $\mathrm{T}(70) 3 \%$ & $0.12(+0)$ & $0.30(+1)$ & $0.10(+2)$ & $0.32(+1)$ & $0.39(-2)$ \\
\hline $\mathrm{T}(70) 15 \%$ & $0.79(-3)$ & $0.46(+3)$ & $0.23(+4)$ & $0.23(+3)$ & $0.18(-4)$ \\
\hline $\mathrm{T}(70) \mathrm{S}(20) 3 \%$ & $0.21(-1)$ & $0.37(+1)$ & $0.30(+2)$ & $0.15(+2)$ & $0.55(+0)$ \\
\hline $\mathrm{T}(70) \mathrm{S}(20) 15 \%$ & $0.30(-1)$ & $0.43(+1)$ & $0.92(+2)$ & $0.23(+2)$ & $0.57(+0)$ \\
\hline $\mathrm{T}(70) \mathrm{S}(50) 3 \%$ & $0.41(-1)$ & $0.23(+1)$ & $0.93(+1)$ & $0.25(+1)$ & $0.12(+0)$ \\
\hline $\mathrm{T}(70) \mathrm{S}(50) 15 \%$ & $0.11(-1)$ & $0.35(+3)$ & $0.18(+4)$ & $0.23(+3)$ & $0.14(-2)$ \\
\hline $\mathrm{S}(0) \mathrm{T}(0) 3 \%$ & $0.12(-1)$ & $0.28(+1)$ & $0.34(+2)$ & $0.18(+2)$ & $0.38(-1)$ \\
\hline $\mathrm{S}(0) \mathrm{T}(28) 0 \%$ & $0.63(-2)$ & $0.33(+1)$ & $0.14(+2)$ & $0.54(+1)$ & $0.34(-1)$ \\
\hline \multicolumn{6}{|c|}{$1.010^{5}$} \\
\hline $\mathrm{T}(0) 3 \%$ & $0.39(-2)$ & $0.82(+1)$ & $0.29(+2)$ & $0.11(+2)$ & $0.19(+0)$ \\
\hline $\mathrm{T}(28) 15 \%$ & $0.35(-2)$ & $0.20(+2)$ & $0.13(+3)$ & $0.13(+2)$ & $0.79(-1)$ \\
\hline $\mathrm{T}(28) 0 \%$ & $0.48(-2)$ & $0.34(+1)$ & $0.11(+2)$ & $0.35(+1)$ & $0.61(-1)$ \\
\hline $\mathrm{T}(28) \mathrm{S}(10) 3 \%$ & $0.23(-1)$ & $0.36(+1)$ & $0.19(+2)$ & $0.78(+1)$ & $0.71(+0)$ \\
\hline $\mathrm{T}(28) \mathrm{S}(10) 15 \%$ & $0.27(-1)$ & $0.33(+1)$ & $0.42(+2)$ & $0.89(+1)$ & $0.11(+1)$ \\
\hline $\mathrm{T}(70) 3 \%$ & $0.81(-2)$ & $0.28(+1)$ & $0.87(+1)$ & $0.22(+1)$ & $0.26(-1)$ \\
\hline $\mathrm{T}(70) 15 \%$ & $0.32(-2)$ & $0.75(+2)$ & $0.46(+3)$ & $0.33(+2)$ & $0.28(-1)$ \\
\hline $\mathrm{T}(70) \mathrm{S}(20) 3 \%$ & $0.20(-1)$ & $0.26(+1)$ & $0.20(+2)$ & $0.90(+1)$ & $0.68(+0)$ \\
\hline $\mathrm{T}(70) \mathrm{S}(20) 15 \%$ & $0.22(-1)$ & $0.25(+1)$ & $0.48(+2)$ & $0.12(+2)$ & $0.11(+1)$ \\
\hline $\mathrm{T}(70) \mathrm{S}(50) 3 \%$ & $0.34(-1)$ & $0.20(+1)$ & $0.83(+1)$ & $0.21(+1)$ & $0.19(+0)$ \\
\hline $\mathrm{T}(70) \mathrm{S}(50) 15 \%$ & $0.25(-1)$ & $0.17(+2)$ & $0.10(+3)$ & $0.12(+2)$ & $0.11(+1)$ \\
\hline $\mathrm{S}(0) \mathrm{T}(0) 3 \%$ & $0.42(-2)$ & $0.35(+1)$ & $0.22(+2)$ & $0.99(+1)$ & $0.11(+0)$ \\
\hline $\mathrm{S}(0) \mathrm{T}(28) 0 \%$ & $0.43(-2)$ & $0.33(+1)$ & $0.11(+2)$ & $0.36(+1)$ & $0.61(-1)$ \\
\hline
\end{tabular}

processing of material). Model $\mathrm{T}(0) 3 \%$ is of a core which is never shocked and in which instantaneous thermal evaporation of grain mantles takes place.

Figures 2 and 3 present the results for Models $\mathrm{S}(0) \mathrm{T}(0) 3 \%$ and $\mathrm{T}(0) 3 \%$. Table 1 gives results for the ratios of some abundances for each of the two models at three different times. At $510^{4}$ years, the differences between the results for models $\mathrm{S}(0) \mathrm{T}(0) 3 \%$ and $\mathrm{T}(0) 3 \%$ are fairly small. The most pronounced differences between val- ues of measurable abundance ratios at $510^{4}$ years for models $\mathrm{S}(0) \mathrm{T}(0) 3 \%$ and $\mathrm{T}(0) 3 \%$ are for the ratios of NS to CS, $\mathrm{SO}$ to $\mathrm{CS}$, and $\mathrm{HCO}$ to $\mathrm{H}_{2} \mathrm{CO}$.

The main cause for the contrasts between the results for $\mathrm{S}(0) \mathrm{T}(0) 3 \%$ and $\mathrm{T}(0) 3 \%$ is the much higher earlytime fractional abundance of atomic oxygen in $\mathrm{T}(0) 3 \%$; $\mathrm{O}$ is a very reactive species which removes many radicals but in shocks is reduced significantly in abundance by the neutral-neutral sequence in which $\mathrm{O}$ and $\mathrm{OH}$ 
Table 2. Abundance ratios at three times (in years) for selected models at the centre of the core corresponding to $A_{V}=13$ mags and $T=275 \mathrm{~K}$

\begin{tabular}{|c|c|c|c|c|c|}
\hline & $\mathrm{HCO} / \mathrm{H}_{2} \mathrm{CO}$ & $\mathrm{SO} / \mathrm{CS}$ & $\mathrm{SO}_{2} / \mathrm{SO}$ & $\mathrm{H}_{2} \mathrm{~S} / \mathrm{CS}$ & $\mathrm{NS} / \mathrm{CS}$ \\
\hline \multicolumn{6}{|c|}{$2.510^{4}$} \\
\hline $\mathrm{T}(0) 3 \%$ & $0.32(-2)$ & $0.35(+2)$ & $0.35(+1)$ & $0.11(+3)$ & $0.51(+0)$ \\
\hline $\mathrm{T}(28) 15 \%$ & $0.35(-2)$ & $0.81(+2)$ & $0.51(+1)$ & $0.91(+2)$ & $0.77(-1)$ \\
\hline $\mathrm{T}(28) 0 \%$ & $0.40(-2)$ & $0.55(+1)$ & $0.42(+1)$ & $0.22(+2)$ & $0.34(-1)$ \\
\hline $\mathrm{T}(28) \mathrm{S}(10) 3 \%$ & $0.37(-1)$ & $0.69(+1)$ & $0.40(+1)$ & $0.23(+2)$ & $0.61(+0)$ \\
\hline $\mathrm{T}(28) \mathrm{S}(10) 15 \%$ & $0.41(-1)$ & $0.97(+1)$ & $0.92(+1)$ & $0.34(+2)$ & $0.31(+0)$ \\
\hline $\mathrm{T}(70) 3 \%$ & $0.34(-2)$ & $0.76(+1)$ & $0.39(+0)$ & $0.17(+2)$ & $0.34(-4)$ \\
\hline $\mathrm{T}(70) 15 \%$ & $0.64(-3)$ & $0.17(+2)$ & $0.20(+1)$ & $0.23(+2)$ & $0.88(-6)$ \\
\hline $\mathrm{T}(70) \mathrm{S}(20) 3 \%$ & $0.17(-1)$ & $0.84(+1)$ & $0.39(+1)$ & $0.27(+2)$ & $0.70(+0)$ \\
\hline $\mathrm{T}(70) \mathrm{S}(20) 15 \%$ & $0.36(-1)$ & $0.22(+2)$ & $0.83(+1)$ & $0.71(+2)$ & $0.74(-1)$ \\
\hline $\mathrm{T}(70) \mathrm{S}(50) 3 \%$ & \multicolumn{5}{|c|}{ Same as $\mathrm{T}(70) 3 \%$} \\
\hline $\mathrm{T}(70) \mathrm{S}(50) 15 \%$ & \multicolumn{5}{|c|}{ Same as $\mathrm{T}(70) 15 \%$} \\
\hline $\mathrm{S}(0) \mathrm{T}(0) 3 \%$ & $0.37(-2)$ & $0.62(+1)$ & $0.34(+2)$ & $0.45(+2)$ & $0.41(-1)$ \\
\hline $\mathrm{S}(0) \mathrm{T}(28) 0 \%$ & $0.45(-2)$ & $0.54(+1)$ & $0.43(+1)$ & $0.22(+2)$ & $0.38(-1)$ \\
\hline \multicolumn{6}{|c|}{$5.010^{4}$} \\
\hline $\mathrm{T}(0) 3 \%$ & $0.29(-2)$ & $0.25(+2)$ & $0.24(+1)$ & $0.45(+2)$ & $0.53(+0)$ \\
\hline $\mathrm{T}(28) 15 \%$ & $0.35(-2)$ & $0.81(+2)$ & $0.51(+1)$ & $0.91(+2)$ & $0.77(-1)$ \\
\hline $\mathrm{T}(28) 0 \%$ & $0.27(-2)$ & $0.77(+1)$ & $0.27(+1)$ & $0.17(+2)$ & $0.15(+0)$ \\
\hline $\mathrm{T}(28) \mathrm{S}(10) 3 \%$ & $0.23(-1)$ & $0.61(+1)$ & $0.36(+1)$ & $0.18(+2)$ & $0.92(+0)$ \\
\hline $\mathrm{T}(28) \mathrm{S}(10) 15 \%$ & $0.32(-1)$ & $0.67(+1)$ & $0.89(+1)$ & $0.22(+2)$ & $0.11(+1)$ \\
\hline $\mathrm{T}(70) 3 \%$ & $0.14(-1)$ & $0.37(+1)$ & $0.30(+1)$ & $0.77(+1)$ & $0.12(-1)$ \\
\hline $\mathrm{T}(70) 15 \%$ & $0.23(-3)$ & $0.44(+4)$ & $0.54(+1)$ & $0.56(+4)$ & $0.91(-4)$ \\
\hline $\mathrm{T}(70) \mathrm{S}(20) 3 \%$ & $0.28(-1)$ & $0.59(+1)$ & $0.44(+1)$ & $0.20(+2)$ & $0.72(+0)$ \\
\hline $\mathrm{T}(70) \mathrm{S}(20) 15 \%$ & $0.31(-1)$ & $0.90(+1)$ & $0.96(+1)$ & $0.32(+2)$ & $0.64(+0)$ \\
\hline $\mathrm{T}(70) \mathrm{S}(50) 3 \%$ & $0.30(-1)$ & $0.34(+1)$ & $0.29(+1)$ & $0.60(+1)$ & $0.27(+0)$ \\
\hline $\mathrm{T}(70) \mathrm{S}(50) 15 \%$ & $0.05(-1)$ & $0.42(+1)$ & $0.15(+4)$ & $0.25(+3)$ & $0.14(-1)$ \\
\hline $\mathrm{S}(0) \mathrm{T}(0) 3 \%$ & $0.05(-1)$ & $0.39(+1)$ & $0.31(+2)$ & $0.23(+2)$ & $0.55(-1)$ \\
\hline $\mathrm{S}(0) \mathrm{T}(28) 0 \%$ & $0.29(-2)$ & $0.76(+1)$ & $0.27(+1)$ & $0.17(+2)$ & $0.16(+0)$ \\
\hline \multicolumn{6}{|c|}{$1.010^{5}$} \\
\hline $\mathrm{T}(0) 3 \%$ & $0.25(-2)$ & $0.19(+2)$ & $0.16(+1)$ & $0.16(+2)$ & $0.44(+0)$ \\
\hline $\mathrm{T}(28) 15 \%$ & $0.22(-2)$ & $0.24(+2)$ & $0.36(+1)$ & $0.13(+2)$ & $0.18(+0)$ \\
\hline $\mathrm{T}(28) 0 \%$ & $0.24(-2)$ & $0.10(+2)$ & $0.16(+1)$ & $0.97(+1)$ & $0.25(+0)$ \\
\hline $\mathrm{T}(28) \mathrm{S}(10) 3 \%$ & $0.13(-1)$ & $0.49(+1)$ & $0.32(+1)$ & $0.11(+2)$ & $0.15(+1)$ \\
\hline $\mathrm{T}(28) \mathrm{S}(10) 15 \%$ & $0.18(-1)$ & $0.39(+1)$ & $0.80(+1)$ & $0.10(+2)$ & $0.21(+1)$ \\
\hline $\mathrm{T}(70) 3 \%$ & $0.41(-2)$ & $0.52(+1)$ & $0.19(+1)$ & $0.52(+1)$ & $0.94(-1)$ \\
\hline $\mathrm{T}(70) 15 \%$ & $0.18(-2)$ & $0.51(+2)$ & $0.43(+1)$ & $0.33(+2)$ & $0.14(+0)$ \\
\hline $\mathrm{T}(70) \mathrm{S}(20) 3 \%$ & $0.16(-1)$ & $0.46(+1)$ & $0.38(+1)$ & $0.13(+2)$ & $0.12(+1)$ \\
\hline $\mathrm{T}(70) \mathrm{S}(20) 15 \%$ & $0.16(-1)$ & $0.41(+1)$ & $0.86(+1)$ & $0.11(+2)$ & $0.22(+1)$ \\
\hline $\mathrm{T}(70) \mathrm{S}(50) 3 \%$ & $0.20(-1)$ & $0.31(+1)$ & $0.28(+1)$ & $0.49(+1)$ & $0.47(+0)$ \\
\hline $\mathrm{T}(70) \mathrm{S}(50) 15 \%$ & $0.22(-1)$ & $0.20(+2)$ & $0.08(+3)$ & $0.23(+2)$ & $0.15(+1)$ \\
\hline $\mathrm{S}(0) \mathrm{T}(0) 3 \%$ & $0.40(-2)$ & $0.37(+1)$ & $0.20(+2)$ & $1.05(+2)$ & $0.48(+0)$ \\
\hline $\mathrm{S}(0) \mathrm{T}(28) 0 \%$ & $0.23(-2)$ & $0.10(+2)$ & $0.16(+1)$ & $0.97(+1)$ & $0.25(+0)$ \\
\hline
\end{tabular}

abstract $\mathrm{H}$ from $\mathrm{H}_{2}$ to form $\mathrm{H}_{2} \mathrm{O}$ (Bergin et al 1998; O'neill $\&$ Williams 1999). For example, SO/CS is about one order of magnitude smaller in the shocked case in which the abundance of free oxygen is much reduced. This result is to be expected: $\sim 70 \%$ of $\mathrm{SO}$ is formed by the reaction $\mathrm{O}+\mathrm{HS}$ and therefore free oxygen is required to form SO from its precursors such as HS. The reduction of the SO formation rate leaves more sulphur available for the production of NS which is facilitated by the fact that even at a temperature of $1000 \mathrm{~K}$ the reaction $\mathrm{N}$ with $\mathrm{H}_{2}$ is slow.

\section{Models with partial freeze-out, time dependent radiative heating and shocks}

We have already seen that some abundance ratios of sulphur-bearing species and of $\mathrm{HCO}$ to $\mathrm{H}_{2} \mathrm{CO}$ are candidate diagnostics of the radiative heating of grains and the hot core dynamical history. We now consider cases in which the radiative heating of the dust and the consequent injection of molecules from the ice into the gas phase, takes place over a finite period of time. 

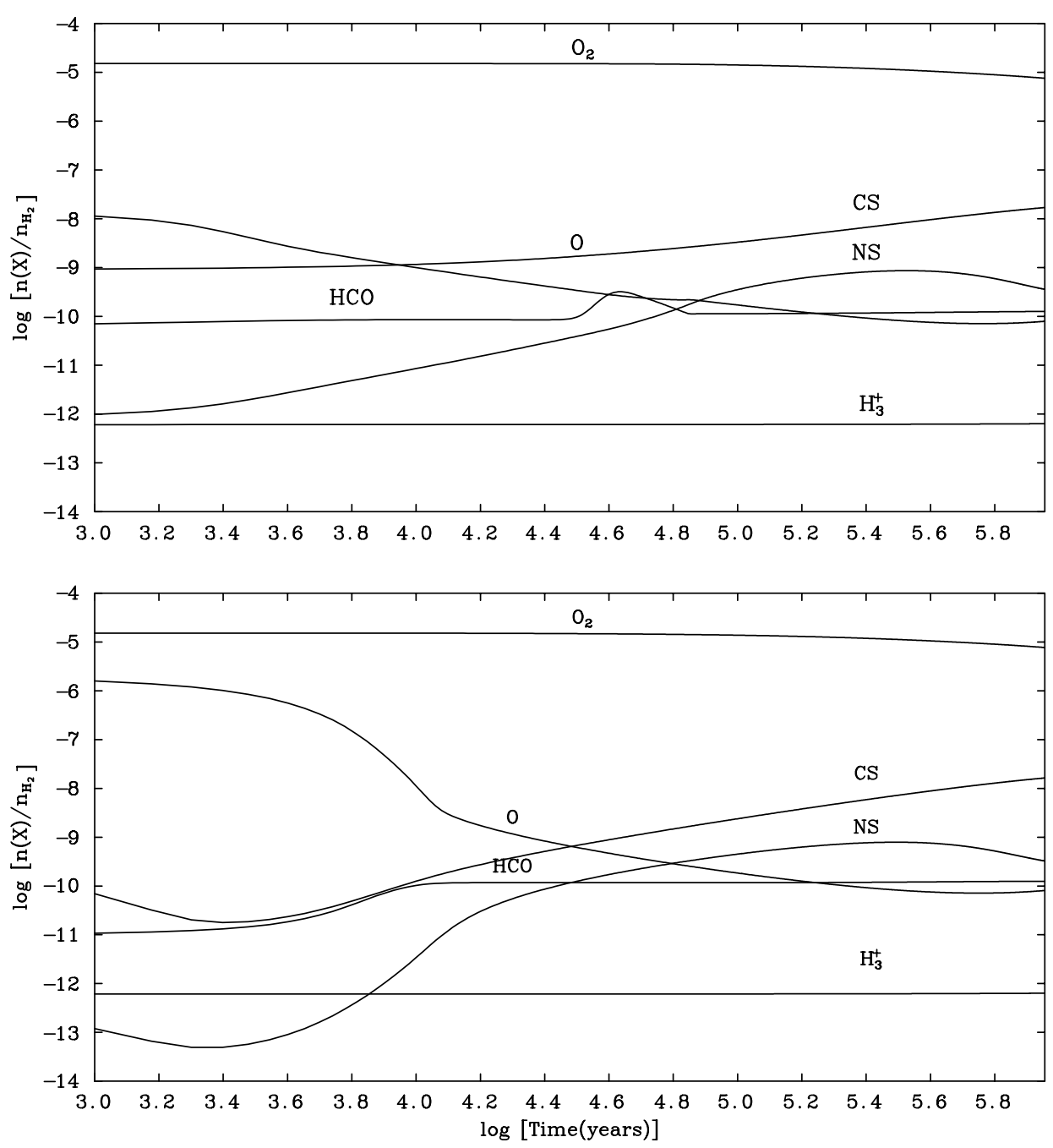

Fig. 2. Evolution of fractional abundances with respect to molecular hydrogen, of species which evaporate at early times for simple models in which freeze-out is incomplete. In each model freeze-out reduces the elemental carbon in gas phase CO to three percent. Top: Results for Model S(0)T(0)3\%. Bottom: Results for Model T(0)3\%

For models $\mathrm{T}(70) \mathrm{S}(20) 15 \%, \mathrm{~T}(70) \mathrm{S}(20) 3 \%$, $\mathrm{T}(70) \mathrm{S}(50) 3 \%, \quad \mathrm{~T}(70) \mathrm{S}(50) 15 \%, \quad \mathrm{~T}(28) \mathrm{S}(10) 3 \% \quad$ and $\mathrm{T}(28) \mathrm{S}(10) 15 \%$, freeze-out after the shock passed was assumed not to take place as grains had already reached temperatures that hindered further freeze-out. As stated in Sect. 2, it is assumed that no second freeze-out phase occurred in model $\mathrm{S}(0) \mathrm{T}(0) 3 \%$; also, no second freeze-out occurred in model $\mathrm{S}(0) 15 \%$. However, in all other models for which a shock was assumed to pass before the onset of radiative heating, a second freeze-out phase was supposed to occur. We terminated this second phase when the same percentage of carbon was left in gas phase species as after the original freeze-out.

Table 1 gives results for abundance ratios for selected computed models at three different times. We concentrate on results for the time of $510^{4}$ years. We do not present the $\mathrm{O} / \mathrm{CO}, \mathrm{H}_{2} \mathrm{O} / \mathrm{O}$, and $\mathrm{O}_{2} / \mathrm{O}$ ratios as those abundance ratios are particularly difficult to measure (note that the SWAS (Bergin et al. 2000) beam is unfortunately much too wide to be of use in observations of a single hot core).
It is evident that the $\mathrm{HCO} / \mathrm{H}_{2} \mathrm{CO}, \mathrm{SO} / \mathrm{CS}, \mathrm{SO}_{2} / \mathrm{SO}$, and NS/CS ratios span particularly large ranges for models at this time. The reasons are exactly the same as stated in Sect. 4.

With the exception of model $\mathrm{T}(70) 3 \%$, the models without shocks have $\mathrm{HCO}$ to $\mathrm{H}_{2} \mathrm{CO}$ abundance ratios of less than $110^{-2}$ at $510^{4}$ years. Model T(70)3\% has a very high $\mathrm{HCO}$ to $\mathrm{H}_{2} \mathrm{CO}$ ratio of 0.12 ; it is the only model without a shock but with slow radiative heating, and high but incomplete depletion. While the presence of a shock in models in which there is less depletion and/or more rapid radiative heating generally leads to a significant increase in the $\mathrm{HCO}$ to $\mathrm{H}_{2} \mathrm{CO}$ ratio, by itself a high $\mathrm{HCO}$ to $\mathrm{H}_{2} \mathrm{CO}$ ratio cannot be used to surmise that a shock has passed.

However, the NS to CS abundance ratio at $510^{4}$ years is much lower in model $\mathrm{T}(70) 3 \%$ than in many models with shocks. Hence, a high NS to CS abundance ratio and a high $\mathrm{HCO}$ to $\mathrm{H}_{2} \mathrm{CO}$ abundance ratio together indicate that a shock occurred. Although we reached this conclusion from abundances calculated at a temperature 

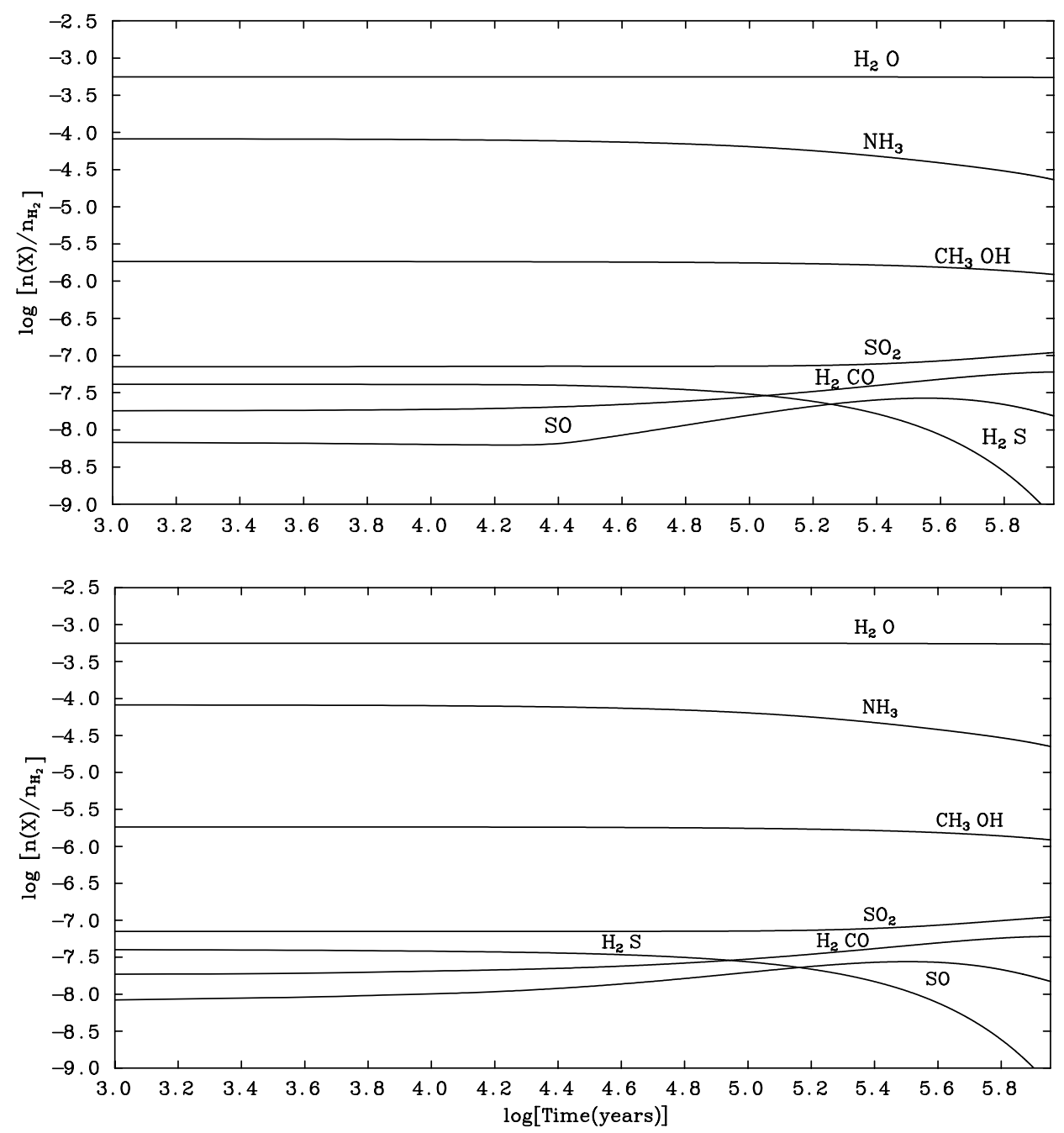

Fig. 3. Evolution of fractional abundances with respect to molecular hydrogen, for species that evaporate at late times for simple models in which freeze-out is incomplete. Top: Results for Model S(0)T(0)3\%. Bottom: Results for Model T(0)3\%

of $\sim 220 \mathrm{~K}$, Table 2 shows that it is still valid at a higher core temperature.

The utility of the abundances of other sulphur-bearing species has been emphasized by previous authors (e.g. Hatchell et al. 1998a), and these species have played a central role in the attempts to derive the "ages" of hot cores. The models with the lowest $\mathrm{CS}$ to $\mathrm{CO}$ ratios at $510^{4}$ years are $\mathrm{T}(28) 15 \%, \mathrm{~T}(70) 15 \%$, and $\mathrm{T}(70) \mathrm{S}(50) 15 \%$, which are also the three models having the highest SO to CS ratios at that time; the $\mathrm{CS}$ to $\mathrm{CO}$ and the SO to $\mathrm{CS}$ ratios depend on how much elemental oxygen is in reactive gas phase species, so it is not surprising that models in which the most material is left in the gas phase at the end of a freeze-out phase show low CS to CO and high SO to CS ratios. The passage of a shock early in the development of a hot core with high remnant gas phase abundances of elements more massive than helium greatly alters these two ratios by causing more oxygen to become contained in $\mathrm{H}_{2} \mathrm{O}$.

In the centre of the core, the three models that have the highest SO to $\mathrm{CS}$ ratios at $510^{4}$ years also have the highest $\mathrm{SO}_{2}$ to $\mathrm{SO}$ ratios at that time. The $\mathrm{SO}_{2}$ to $\mathrm{SO}$ ra- tio is reduced if a shock occurs fairly early in a hot core's evolution. Those same three models show the highest $\mathrm{H}_{2} \mathrm{~S}$ to CS ratios. This result is consistent with CS being removed to form $\mathrm{SO}$ in reactions with $\mathrm{O}$ sufficiently rapidly that the $\mathrm{CS} / \mathrm{CO}$ ratio is kept low.

\section{Conclusions}

As found previously (Hatchell et al. 1998a), the fractional abundances of various sulphur-bearing species depend rather sensitively on depletions, hot core "age", and grain thermal history. The work presented here shows that the abundance ratios of $\mathrm{HCO} / \mathrm{H}_{2} \mathrm{CO}$ and $\mathrm{NS} / \mathrm{CS}$ seem particularly well suited to infer whether a shock has passed through a hot core. However, they are probably useful in this way only if freeze-out during the pre-stellar phase is not total.

It should be noted that we have made some rather strong assumptions about the role of surface reactions in the chemistry of the hot cores. We have assumed that hydrogenation of species to form saturated hydrocarbons dominates on grain surfaces. In fact, the use of an 
approach like that of Caselli et al. (1998) might well lead us to conclude that the grain surface reactions lead to far richer surface compositions than we have supposed. We have also assumed that the shock is not powerful enough to destroy the refractory material. The release of the latter, however, would not change the conclusion of this study as the silicon released should not significantly affect the $\mathrm{NS} / \mathrm{CS}$ nor the $\mathrm{HCO} / \mathrm{H}_{2} \mathrm{CO}$ ratios.

At a fractional abundance of $\sim 10^{-10}$, HCO is probably too weak to be detectable other than in the $(3 \mathrm{~mm})$ ground state transitions, even in Orion and Sgr-B2 (Turner 1989) but measurements of the NS to CS abundance ratios in hot cores should be made. NS is not a commonly observed species but detections of NS emission towards a number of hot cores were reported by Hatchell et al. (1998b): their observations of NS and CS give ratios close to $\sim 0.1$. The hot cores in their sample are believed to be all at the same evolutionary stage as the authors did not find a large variation in their chemistry. Also, McGonagle \& Irvine (1997) reported an extensive survey for nitrogen sulfide in Giant Molecular Clouds (GMC). They found that its fractional abundance is of the order of few $10^{-10}$. In general, this is consistent with the estimates of our models. Their estimate for the column density of NS is, however, too uncertain to substantiate our conclusions that the CS/NS ratio could be a good indicator of shocks in hot cores: their observations were performed with spatial resolution of 86 , 45 and 35 arcsec for observations of NS $J=3 / 2 \Rightarrow 1 / 2$, NS $J=5 / 2 \Rightarrow 3 / 2$ and NS $J=7 / 2 \Rightarrow 5 / 2$ respectively, and they conclude that much higher spatial resolution observations of NS are indeed needed. Comparisons should be made with high resolution (interferometric) observations for a large sample of hot cores where a resolution of about 1 arcsec (necessary even for the nearest hot core) can be achieved. Indeed, the low rotational and excitation temperatures deduced from their observations suggest that NS emission arises from extended material of relatively modest density $\left(<\sim 10^{5} \mathrm{~cm}^{-3}\right)$.

Acknowledgements. SV acknowledges the financial support of PPARC. DAW is grateful to PPARC for the award of a Senior Fellowship. PC acknowledges support from the ASIgrants ARS-98-116 and ARS-78-1999, and from the MURST research program "Dust and Molecules in Astrophysical Environments". The authors thank A. G. Gibb and M. G. Hoare for useful discussions.

\section{References}

Bachiller, R. 1996, ARA\&A, 34, 111

Bergin, E. A., et al. 2000, ApJ, 539, L129

Bergin, E. A., Melnick, G. J., \& Neufeld, D. A. 1998, ApJ, 499, 777

Bergin, E. A., Goldsmith, P. F., Snell, R. L., \& Langer, W. D. 1997, ApJ, 482, 285
Brown, P. D., Charnley, S. B., \& Millar, T. J. 1988, MNRAS, 231, 409

Caselli, P., Hasegawa, T. I., \& Herbst, E. 1993, ApJ, 408, 548

Caselli, P., Hasegawa, T. I., \& Herbst, E. 1998, ApJ, 495, 309

Caselli, P., Walmsley, C. M., Tafalla, M., Dore, L., \& Myers, P. C. 1999, ApJ, 523, L165

Ceccarelli, C., Loinard, L., Castets, A., Tielens, A. G. G. M., \& Caux, E. 2000, A\&A, 357, L9

Cesaroni, R., Hofner, P., Walmsley, C. M., \& Churchwell, E. 1998, A\&A, 331, 709

Cesaroni, R., Felli, M., Jenness, T., et al. 1999, A\&A, 345, 949

Charnley, S. B. 1997, ApJ, 481, 396

Charnley, S. B., Tielens, A. G. G. M., \& Millar, T. J. 1992, ApJ, 399, L71

Gibb, A. G., \& Little, L. T. 1998, MNRAS, 295, 299

Hanson, M. M. 1998, in Boulder-Munich II: Properties of Hot, Luminous Stars, ed. I. Howarth, ASP Conf. Ser., 131, 1

Hatchell, J., Thompson, M. A., Millar, T. J., \& MacDonald, G. H. 1998a, A\&A, 338, 713

Hatchell, J., Thompson, M. A., Millar, T. J., \& MacDonald, G. H. 1998b, A\&AS, 133, 29

Kramer, C., Alves, J., Lada, C. J., et al. 1999, A\&A, 342, 257

Kurtz, S., Cesaroni, R., Churchwell, E., Hofner, P., \& Walmsley, C. M. 2000, in Protostars and Planets IV, ed. V. Mannings, A. P. Boss, \& S. S. Russell (University of Arizona Press), 299

Lada, C. J., Oppenheimer, M., \& Hartquist, T. W. 1978, ApJ, 226, L153

McGonagle, D., \& Irvine, W. M. 1997, ApJ, 477, 711

Millar, T. J. 1993, in Dust and Chemistry in Astronomy, ed. T. J. Millar, \& D. A. Williams (Bristol, Institute of Physics Publishing), 143

Millar, T. J., Farquhar, P. R. A., \& Willacy, K. 1997, A\&AS, 121,139

Millar, T. J., \& Hatchell, J. 1998, Faraday Discussions 109, 15

Millar, T. J., Macdonald, G. H., \& Gibb, A. G. 1997, A\&A, 325,1163

Molinari, S., Testi, L., Brand, J., Cesaroni, R., \& Palla, F. 1998, ApJ, 505, L39

O’Neill, P. T., \& Williams, D. A. 1999, AP\&SS, 266, 539

Oppenheimer, M., \& Dalgarno, A. 1974, ApJ, 187, 231

Reid, M. J., Argon, A. L., Masson, C. R., Menten, K. M., \& Moran, J. M. 1995, ApJ, 443, 238

Rowan-Robinson, M. 1980, ApJS, 44, 403

Ruffle, D. P., Hartquist, T. W., Caselli, P., \& Williams, D. A. 1999, MNRAS, 306, 691

Shepherd, D. S., \& Churchwell, E. 1996, ApJ, 472, 225

Turner, B. E. 1989, ApJS, 70, 539

van Dishoeck, E. F. 1998, in The Molecular Astrophysics of Stars and Galaxies, ed. T. W. Hartquist, \& D. A. Williams (Oxford, Clarendon Press), 53

Viti, S., \& Williams, D. A. 1999, MNRAS, 305, 755

Walmsley, C. M., \& Schilke, P. 1993, in Dust and Chemistry in Astronomy, ed. T. J. Millar, \& D. A. Williams (Bristol, Institute of Physics Publishing), 37

Willacy, K., Langer, W. D., \& Velusamy, T. 1998, ApJ, 507, L171

Williams, D. A. 1993, in Dust and Chemistry in Astronomy, ed. T. J. Millar, \& D. A. Williams (Bristol, Institute of Physics Publishing), 143 